\title{
Nouveaux enjeux des maladies à transmission vectorielle pour les villes canadiennes
}

\author{
Luka $\mathrm{N}^{1 *}$
}

\author{
Affiliation \\ ${ }^{1}$ Écoles d'architecture et d'urbanisme, Université McGill, Montréal (Québec) \\ *Correspondance : nik.luka@mcgill.ca
}

Citation proposée : Luka N. Nouveaux enjeux des maladies à transmission vectorielle pour les villes canadiennes. Relevé des maladies transmissibles au Canada 2016;42:241-2. https://doi.org/10.14745/ccdr.v42i10a12f

\section{Contexte}

Au Canada, les questions de la résilience, de la solidité et de la santé ont pris beaucoup d'importance dans les débats sur la construction des villes au cours des dernières années. Le changement climatique et l'augmentation des maladies chroniques occupent désormais une place centrale dans les débats sur la politique urbaine. Les urbanistes, les architectes et les autres spécialistes de l'environnement bâti communiquent maintenant plus ouvertement avec leurs homologues dans d'autres disciplines, mais on en sait relativement peu sur ce que signifient les nouveaux enjeux associés aux maladies à transmission vectorielle (MTV) pour la conception des villes et I'urbanisme au Canada.

\section{Objectif}

Synthèse des principaux thèmes soulevés lors d'un atelier sur les MTV dans les villes canadiennes du point de vue de la conception des villes et de l'urbanisme, qui a eu lieu à I'Université McGill en avril 2016.

\section{Narratif}

Pendant cet atelier sur deux jours, des experts de nombreux domaines importants pour la santé publique (notamment psychiatrie, épidémiologie, zoonotique, parasitologie, microbiologie, immunologie, écologie du paysage, géographie, philosophie politique, urbanisme et génie civil) ont partagé des réflexions sur des questions très diverses qui se recoupent de façon complexe. Ce qui suit est une brève description des questions (et des implications) concernant la façon dont les régions métropolitaines au Canada sont planifiées, conçues, construites et entretenues, ainsi que les répercussions potentielles sur la propagation des MTV. Ces questions s'articulent autour de cinq points clés à développer ultérieurement.

Il est absolument nécessaire d'approfondir la discussion interdisciplinaire et intersectorielle sur les maladies infectieuses à transmission vectorielle et sur les affections chroniques, car elles touchent la population canadienne dans les villes, les banlieues et les zones périurbaines, où la grande majorité des 36 millions d'habitants du Canada passent leur vie au quotidien (1). Au moment où tous les échelons de gouvernement s'embarquent dans des projets ambitieux de renouvellement des infrastructures - en particulier dans les paysages d'après-guerre vieillissants qui constituent l'essentiel de nos régions métropolitaines -, il est de nouveau nécessaire que les responsables des politiques, les urbanistes et les représentants élus (qui contrôlent les budgets publics et les priorités pour la mise en œuvre) entament des échanges détaillés, axés sur les actions, sur ce qui peut être fait. Les nouveaux enjeux associés aux MTV doivent être abordés, tout comme les affections chroniques liées aux modes de vie sédentaires, qui ont attiré une attention considérable au cours des vingt dernières années, depuis que nous avons pris davantage conscience des facteurs contextuels " en amont " qui influent sur la santé publique - tels que la forme urbaine qui oblige les usagers à être dépendants de la voiture pour leurs activités quotidiennes.

Les compromis avec d'autres considérations sociales, économiques et culturelles doivent être identifiés et discutés. Des contradictions surgissent entre les objectifs, les résultats souhaitables et les stratégies que nous pouvons entreprendre d'une façon qui soit à la fois opportune et faisable (sur le plan social et politique). Certaines tensions sont apparentes entre ce qui peut facilement être fait pour remédier aux maladies chroniques - qui, pour beaucoup, sont liées à la configuration physique de nos régions métropolitaines, par exemple à la forme urbaine, à la densité et au " potentiel piétonnier » $(2,3)$ - et ce qui peut être fait pour lutter contre les MTV, lesquelles sont souvent exacerbées par les diverses sortes de mesures de médiation prises pour faire face aux maladies chroniques. Par exemple, il est connu que le potentiel piétonnier augmente lorsque les personnes et les activités sont comprimées dans l'espace (à savoir, la densité), mais cela nécessite ensuite une augmentation de la biomasse (c'est-à-dire les arbres et autres formes de végétation) pour compenser les effets d'îlot thermique urbain et le malaise psychologique ressenti par de nombreuses personnes dans les quartiers surpeuplés et à forte densité de construction. La gestion sur place des eaux pluviales (p. ex. bassins de rétention) est désormais considérée comme souhaitable dans les contextes urbains et suburbains pour éviter de faire subir une pression inutile aux écosystèmes et aux réseaux $d$ 'assainissement, mais les tiques et les moustiques (vecteurs connus de la maladie de Lyme et du virus du Nil occidental, respectivement) pourraient ainsi avoir largement la possibilité de proliférer. II s'agit là de questions fondamentales de résilience et de conception adaptative qui ont été soulevées dans d'autres domaines de la planification (4-6). 
Les problèmes d'échelle (variabilité d'échelle) et la résolution de l'information à la disposition des personnes clés à différents maillons de la " chaîne " allant des preuves aux politiques et à la mise en pratique sont importants. Comment devons-nous repérer, analyser et comprendre les problèmes? Comment pouvons-nous ensuite élaborer et mettre en œuvre des interventions efficaces compte tenu des contraintes existantes? Comment devons-nous surveiller et ajuster les diverses stratégies d'intervention? Au centre des préoccupations, on retrouve plus précisément la question du « qui fait quoi » (différents échelons de gouvernement), la qualité et la continuité des données et la connaissabilité (c'est-à-dire la solidité des modèles utilisés pour prédire les résultats dans différentes conditions).

Comment composer avec les limites réelles (pratiques) de la planification et de la politique, en particulier dans les démocraties libérales? La lutte contre les MTV et les affections chroniques dans les régions métropolitaines constitue ce que les scientifiques politiques appellent un « problème vicieux ». Parmi les thèmes particuliers qui ont été évoqués pendant l'atelier figurent la confiance du public, la nature de l'autorité et du contrôle, et la qualité, l'efficacité et la rapidité de la prise de décision dans les démocraties délibératives.

Pour finir, une question : quelles sortes de mécanismes pouvons-nous mettre au point pour assurer la continuité et l'efficacité de stratégies adaptées à des problèmes dont l'importance fluctue au fil du temps? Trois points sont à noter ici : premièrement, la production et la diffusion ou le transfert des connaissances, c'est-à-dire comment sensibiliser davantage les non-spécialistes et, surtout, les différents groupes de spécialistes dans divers secteurs liés à la santé publique, aux politiques et aux affaires urbaines; deuxièmement, comment surmonter les vases clos qui ont tendance à se développer au sein des secteurs de politique et de pratique et entre eux; et troisièmement, revoir la distribution des responsabilités entre les échelons de gouvernance au Canada, étant donné que les municipalités sont obligées de trouver un équilibre entre des objectifs contradictoires par le biais de la planification (y compris notamment l'aménagement du territoire pour maintenir leur assiette fiscale, qui finance un large éventail de services et d'infrastructures), mais n'ont toutefois que peu de pouvoir pour ce qui est d'exercer un véritable contrôle sur l'aménagement du territoire et de garantir des budgets de fonctionnement adéquats au moyen des impôts. Le sujet des MTV met en lumière la difficulté, insoluble à ce jour, à trouver un équilibre entre subsidiarité et suprématie (sur le plan juridique) ou centralisation (sur le plan institutionnel).

\section{Conclusion}

Les tensions et les enjeux révélés entre les différents buts et objectifs politiques ne sont pas nouveaux au sens large - des problèmes semblables ont été abordés par Agyeman (7),
Fainstein (8) et dans deux nouveaux recueils édités par Dale et al. (9) et par Reed et Lister (10). Les échanges entre les participants de l'atelier ont permis de suggérer divers chemins à suivre et de repérer des lacunes importantes qui doivent impérativement être comblées. En particulier, un symposium structuré pourrait être organisé, en prévision duquel les invités prépareraient des prises de position détaillées et dans le cadre duquel un ordre du jour serait élaboré pour la recherche approfondie, ce qui pourrait mener à des demandes ciblées en matière de recherche interdisciplinaire concertée.

\section{Conflit d'intérêts}

Aucun.

\section{Références}

1. Gordon DLA, Janzen M. Suburban nation? Estimating the size of Canada's suburban population. J Archit Plann Res 2013;30(3):197-220.

2. Ewing R, Handy S. Measuring the unmeasurable: urban design qualities related to walkability. J of Urban Des 2009;14(1):65-84.

3. Manaugh K, El-Geneidy AM. Validating walkability indices: how do different households respond to the walkability of their neighborhood? Transp Res D Transp Environ 2011:16(4):309-15.

4. Burton P, Mustelin J. Planning for climate change: is greater public participation the key to success? Urban Policy Res 2013;31(4):399-415.

5. Lister N-M. Resilience: designing the new sustainability. Topos, 90 (Special issue: Resilient Cities and Landscapes) 2015a:14-21.

6. Lister N-M, Brocki M, Ament R. Integrated adaptive design for wildlife movement under climate change. Front Ecol Environ 13(9) 2015b:493-502. doi:10.1890/150080.

7. Agyeman J. Introducing just sustainabilities: policy, planning and practice. London: Zed Books / Palgrave Macmillan; 2013.

8. Fainstein SS. Can we make the cities we want? In Beauregard RA, Body-Gendrot S, editors. The urban moment. Thousand Oaks CA: Sage; 1999. p. 249-72.

9. Dale A, Dushenko WT, Robinson P, editors. Urban sustainability: reconnecting space and place. Toronto: University of Toronto Press; 2014.

10. Reed C, Lister N-M, editors. Projective ecologies. New York: ACTAR; 2014. 